\title{
Patterns of neurocognitive impairment in first- episode bipolar disorder and schizophrenia
}

\author{
Suzanne L. Barrett, Ciaran C. Mulholland, Stephen J. Cooper and Teresa M. Rushe
}

\section{Background}

Researching psychotic disorders in unison rather than as separate diagnostic groups is widely advocated, but the viability of such an approach requires careful consideration from a neurocognitive perspective.

\section{Aims \\ To describe cognition in people with bipolar disorder and schizophrenia and to examine how known causes of variability in individual's performance contribute to any observed diagnostic differences.}

\section{Method}

Neurocognitive functioning in people with bipolar disorder $(n=32)$, schizophrenia $(n=46)$ and healthy controls $(n=67)$ was compared using analysis of covariance on data from the Northern Ireland First Episode Psychosis Study.

\section{Results}

The bipolar disorder and schizophrenia groups were most impaired on tests of memory, executive functioning and language. The bipolar group performed significantly better on tests of response inhibition, verbal fluency and callosal functioning. Between-group differences could be explained by the greater proclivity of individuals with schizophrenia to experience global cognitive impairment and negative symptoms.

\section{Conclusions}

Particular impairments are common to people with psychosis and may prove useful as endophenotypic markers. considering the degree of individuals' global cognitive impairment is critical when attempting to understand patterns of selective impairment both within and between these diagnostic groups.

\section{Declaration of interest}

None.
Neurocognitive impairment is recognised as a core feature of schizophrenia, and specific deficits (e.g. memory impairments) have been deemed potentially useful as endophenotypic markers for this disorder. ${ }^{1}$ A similar but less severe pattern of cognitive impairment has also been observed in people with bipolar disorder $^{2}$ and in people with psychotic depression. ${ }^{3}$ These similarities between diagnostic groups are also found in genetic studies, which show an overlap in genetic susceptibility to both schizophrenia and bipolar disorder. ${ }^{4}$ Epidemiological and developmental observations of commonalities are also apparent, such as an excess of winter-spring births, abnormal dermatoglyphs, an excess of perinatal complications and an increased cross-risk for schizophrenia and bipolar disorder in affected families. ${ }^{5}$ This convergence from multiple disciplines challenges Kraepelin's categorisation of the psychoses into schizophrenia and bipolar disorder and argues for their study across traditional nosological boundaries.

It remains common practice in neurocognitive studies to separate people with psychosis on the basis of their diagnosis for comparative purposes, or to restrict study entry to individuals with a schizophrenia-spectrum diagnosis. However, it can be argued that cognitive differences between affective and nonaffective groups as currently defined are largely quantitative rather than qualitative, and/or attributable to variation in factors other than psychosis such as negative symptoms and global cognitive impairment. Hence, diagnostic categorisation may serve only to compromise the statistical power of studies, undermining efforts to elucidate the aetiology and pathophysiology of psychosis. The following analyses represent a first step in considering the utility of Kraepelin's dichotomy for neurocognitive research purposes using psychological data derived from people participating in the Northern Ireland First Episode Psychosis Study (NIFEPS) The primary aims were to describe cognitive functioning in participants with bipolar disorder and schizophrenia at first episode and to examine how known causes of variability in neurocognitive functioning contribute to any observed diagnostic differences.

\section{Method}

Participants in NIFEPS were incident cases recruited via referral from psychiatric services between January 2003 and December 2004 in the Belfast and Northern Health and Social Service Area of Northern Ireland. Ethical approval was obtained from the Office of Research Ethics Committees Northern Ireland. Prior to study entry, participants gave written consent after they had been provided with a full explanation of what the study entailed. All participants were aged 18-64 at the time of recruitment.

\section{Participants}

The 78 participants with first-episode psychosis included in the following analyses satisfied ICD- $10^{6}$ criteria for schizophrenia $(n=44)$, schizoaffective disorder $(n=2)$ and bipolar disorder/ mania $(n=32)$ as determined by clinical consensus reviews of patient histories and structured interviews using the Schedule for Clinical Assessment in Neuropsychiatry (SCAN). ${ }^{7}$ The majority $(91 \%)$ were being prescribed antipsychotics at the time of testing (5\% typical antipsychotics). Healthy comparison participants $(n=67)$ were recruited from general practitioner surgeries and by advertising on the Belfast City Hospital site. Controls were selected to be similar to participants in the schizophrenia and the bipolar disorder groups on distributions of gender and age.

\section{Exclusion criteria}

A total of 141 NIFEPS participants agreed to undergo neurocognitive testing. General exclusion criteria applied to this cohort 
were: evidence of psychosis resulting from a clear organic aetiology $(n=3)$, the subsequent identification of a previous episode of psychosis $(n=1)$ and scoring $<70$ on IQ measurement $(n=24)$. The relative composition of this sample in terms of diagnosis remained largely unchanged by this process of exclusion (the schizophrenia group originally comprised $42.3 \%$ of the sample and this became $40.7 \%$; the bipolar disorder group were $24.1 \%$ of the sample and this became $28.3 \%$ ). The remaining individuals were excluded from these analyses because they met ICD-10 criteria for psychotic depression $(n=7)$, alcohol-related psychosis $(n=4)$, drug-induced psychosis $(n=11)$, delusional disorder $(n=3)$ and psychosis not otherwise specified $(n=10)$. Controls were screened for psychotic disorders using the Psychosis Screening Questionnaire. ${ }^{8}$ None of the controls reported a chronic neurological illness, current substance use disorder or a history of substance dependence. Three were excluded because they scored $<70$ on IQ measurement.

\section{Procedure}

Assessments were undertaken within 3 months of the individual's first presentation to psychiatric services (median 38 days). Eightysix per cent of people were in hospital at the time of testing: less than $5 \%$ of the incident cases of psychosis identified by NIFEPS were treated in the community for their first episode. To ensure that concurrent clinical data would be available, testing occurred at the first date agreed by the participant after clinical interview. Symptoms were assessed using the Positive and Negative Syndrome Scale (PANSS) ${ }^{9}$ and the Beck Depression Inventory (BDI). ${ }^{10}$

The neurocognitive battery measured a number of neuropsychological domains: global intellectual functioning (Wechsler Abbreviated Scale of Intelligence (WASI) two-test version: vocabulary and matrix reasoning); ${ }^{11}$ premorbid IQ (National Adult Reading Test (NART)); ${ }^{12}$ verbal attention and working memory (Digit Span Forward/Backward from the Wechsler Memory Scale) ${ }^{13}$ spatial attention (Corsi Block-tapping Test); ${ }^{14}$ verbal memory (paired associates from the Wechsler Memory Scale) $;^{13}$ visual memory (Rey Osterrieth Complex Figure Test with a recall trial at $30 \mathrm{~min}) ;{ }^{15}$ response inhibition and attentional setshifting (Hayling and Brixton Tests); ${ }^{16}$ verbal fluency (Controlled Oral Word Association (COWA) test); ${ }^{17}$ language (Perin's
Spoonerisms); ${ }^{18}$ and callosal function (The Tactile Finger Localisation Test). ${ }^{19}$

\section{Statistical analysis}

Data analysis was conducted using SPSS V.11.5 (SPSS Inc., 2002; PC version). Neuropsychological variables were assessed for normality (skewing and kurtosis). Extreme values were identified using boxplots and truncated to three standard deviations from the mean. To reduce positive skewing, a square root transformation was applied to Hayling error scores and participants' ages were $\log (\ln )$ transformed. Missing data points for neuropsychological data were then imputed ${ }^{20}$ using NORM V.2.03, PC version $(m=3)$. The resulting test scores were standardised to the performance of the control group ( $z$ scored) and error scores were sign changed to provide a standard metric for comparison across tests.

Analysis of covariance (ANCOVA) was used to examine differences in test performance, with group (schizophrenia, bipolar disorder and controls) as a between-participants factor and test scores entered as dependent variables, with age entered as a covariate where it contributed to the model (groups differed significantly on this demographic variable). Post hoc Bonferronicorrected pair-wise comparisons were used to detail group differences. Significant differences on clinical and general cognitive performance variables were then identified to determine potential explanatory covariates for consideration in further analyses. Descriptive statistics for these variables are shown in Table 1.

The influence of potential explanatory covariates for group differences (i.e. those variables on which groups differed) were examined sequentially in a series of ANCOVAs to determine the extent to which each of these factors contributed to the neurocognitive differences observed between the groups. Given justifiable concerns about covarying for IQ where IQ impairment is considered intrinsic to a disorder, we then examined the effect of a minimal $v$. a sizeable global cognitive impairment on the overall shape of the schizophrenia group's neurocognitive profile relative to the bipolar disorder group, who showed preserved IQ. A 'low IQ' schizophrenia group and a 'preserved IQ' schizophrenia group were created for this purpose using a median split of the current IQ variable in people with schizophrenia

\begin{tabular}{|c|c|c|c|c|c|c|}
\hline & Controls & Bipolar disorder & Schizophrenia & $\begin{array}{l}\text { 'Preserved' IQ } \\
\text { schizophrenia }\end{array}$ & $\begin{array}{c}\text { 'Low' IQ } \\
\text { schizophrenia }\end{array}$ & Comparisons \\
\hline Total, $n$ & 67 & 32 & 46 & 23 & 23 & \\
\hline Gender, male: $n$ (\%) & $39(58.20)$ & $16(50.00)$ & $32(69.60)$ & $15(65.2)$ & $17(73.9)$ & \\
\hline Age, years: mean (s.d.) & $33.19(10.95)$ & $36.71(9.31)$ & $28.99(9.92)$ & $26.02(6.19)$ & $31.97(12.01)$ & $\begin{array}{c}\mathrm{SCZ}<\mathrm{BPD}, * * \\
\text { preserved } \mathrm{IQ}<\text { low } \mathrm{IQ} *\end{array}$ \\
\hline WASI IQ, mean (s.d.) & $105.72(11.28)$ & $98.81(16.81)$ & $88.87(13.00)$ & $98.35(11.68)$ & $79.39(4.66)$ & $\begin{array}{l}\text { controls }>\mathrm{SCZ},{ }^{* *} \\
\text { controls }>\mathrm{BD},{ }^{*} \mathrm{BD}>\mathrm{SCZ},{ }^{* *} \\
\text { preserved } \mathrm{IQ}>\mathrm{IOW} \mathrm{IQ}{ }^{\star *}\end{array}$ \\
\hline Premorbid IQ, mean (s.d.) & $107.74(9.98)$ & $103.29(11.88)$ & $98.58(11.61)$ & $102.25(12.21)$ & $94.95(9.54)$ & $\begin{array}{l}\text { controls }>\mathrm{SCZ}{ }^{* *} \\
\text { controls }>\mathrm{BD}^{*} \\
\text { preserved } \mathrm{IQ}>\mathrm{IOW} \mathrm{IQ}^{*}\end{array}$ \\
\hline \multicolumn{2}{|c|}{ PANSS total positive, mean (s.d.) } & $17.39(5.51)$ & $17.70(6.06)$ & $18.09(5.21)$ & $17.32(6.88)$ & \\
\hline \multicolumn{2}{|c|}{ PANSS excitement-mania, mean (s.d.) ${ }^{\mathrm{b}}$} & $6.09(1.67)$ & $5.96(2.34)$ & $5.78(2.11)$ & $6.13(2.59)$ & \\
\hline \multicolumn{2}{|c|}{ PANSS total negative, mean (s.d.) } & $9.81(3.25)$ & $15.65(5.1)$ & $14.81(4.58)$ & $16.45(5.54)$ & $\mathrm{SCZ}>\mathrm{BD} * *$ \\
\hline \multicolumn{2}{|c|}{ BDI, mean (s.d.) } & $9.97(10.83)$ & $17.04(10.29)$ & $16.14(10.07)$ & $17.23(11.07)$ & $\mathrm{SCZ}>\mathrm{BD} * *$ \\
\hline \multicolumn{7}{|c|}{ 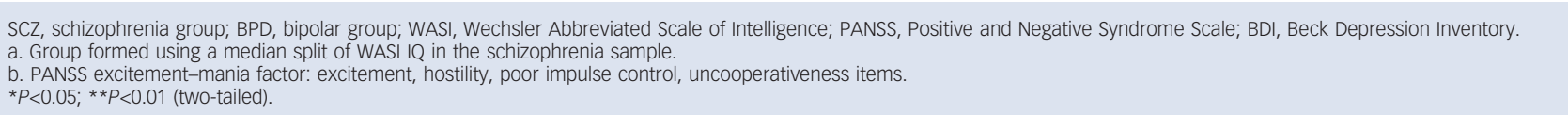 } \\
\hline
\end{tabular}


(median 87, producing a split of the schizophrenia group at approximately 1 standard deviation below the expected average IQ for the general population). Neurocognitive performance according to these 'preserved' and 'low' IQ designations were then plotted and differences between groups were again compared using ANCOVA (age entered as a covariate where it contributed to the model).

Finally, as the covariates WASI IQ, negative symptoms and depression were correlated in this sample (IQ and negative symptoms, $r=-0.3, P<0.01$; IQ and depression, $r=-0.18$, $P=0.06$; depression and negative symptoms, $r=0.32, P<0.01$ ), multiple regression was used to determine the unique variance in neurocognitive performance attributable to each of the factors considered in these analyses.

\section{Results}

The schizophrenia group was significantly younger than the bipolar disorder group $(F(2,142)=6.37, P<0.01$; schizophrenia $<$ bipolar disorder, $P<0.01)$. Both the bipolar disorder and schizophrenia groups had a lower current IQ $(F(2,142)=22.19$, $P<0.001)$ and premorbid IQ $(F(2,142)=10.25, P<0.001)$ than the control sample, but differed from each other on current IQ only (Table 1). In terms of symptomatology, the bipolar disorder and schizophrenia groups had similar mean positive symptom scores and were also similar on an 'excitement-mania' factor extracted from the PANSS. ${ }^{21}$ However, the schizophrenia group had higher mean BDI $(t(76)=2.92, P<0.01)$ and PANSS negative scores $(t(76)=6.43, P<0.001)$.

Table 2 shows the mean test scores for the schizophrenia and bipolar disorder groups, as well as the groups' $z$-scores (adjusted for age), and ANCOVA results from the primary analysis. ANCOVAs showed group as a significant factor for nine of the ten measures (not significant for verbal attention). Post hoc tests showed that, relative to controls, the schizophrenia group was significantly impaired on eight of the ten measures $(P<0.01)$, the exceptions being verbal attention (not significant) and visual attention $(P<0.05)$. The bipolar disorder group was significantly impaired on six of the ten measures relative to controls $(P<0.01)$ : working memory, verbal and visual memory, executive set shifting, verbal response inhibition and language functioning.
Unlike the schizophrenia group, the bipolar disorder group was unimpaired on measures of attention, verbal fluency and callosal functioning.

The schizophrenia group had significantly lower $z$-scores relative to the bipolar disorder group on three measures in post hoc comparisons: verbal response inhibition $(P<0.05)$, verbal fluency $(P<0.01)$ and callosal function $(P<0.05)$. In an attempt to account for these differences, we first considered the potential influence of variations in negative and depressive symptoms, both of which were higher in the schizophrenia group (Table 1), using 'patient only' post hoc ANCOVAs. Patient group differences in response inhibition and verbal fluency reached trend levels of significance after controlling for depression (response inhibition, $P=0.05$; verbal fluency, $P=0.09$ ) but were not significant when only negative symptoms were controlled for (response inhibition, $P=0.64$; verbal fluency, $P=0.73$ ). Neither depression nor negative symptoms contributed to the ANCOVAs that compared callosal functioning in the schizophrenia and bipolar groups.

Meanwhile, all three groups differed significantly on measures of IQ. Hence, the primary analyses comparing test scores in schizophrenia, bipolar disorder and control groups were repeated with current IQ entered as an additional covariate in the ANCOVAs (Table 2). Differences between the controls and the schizophrenia and bipolar disorder groups reduced in magnitude but in general, remained significant: visual attention in the schizophrenia group was an exception (rendered non-significant). However, the schizophrenia and bipolar disorder groups no longer significantly differed on any measure in these analyses. In order to examine the effect of IQ with reference to diagnosis, graphs were plotted to show the neurocognitive profile of individuals when designated to one of the following three groups: schizophrenia 'low' IQ, schizophrenia 'preserved' IQ or bipolar disorder (Fig. 1). Group characteristics are shown in Table 1.

The 'preserved' IQ schizophrenia group had a similar mean IQ and a similarly shaped neurocognitive profile to the bipolar disorder group: multiple comparisons indicated that they did not significantly differ on any measure, including premorbid IQ. However, the profile of the 'low' IQ schizophrenia group was noticeably different. Group comparisons showed that the areas of greatest divergence between the 'low' IQ schizophrenia group and the other two groups ('preserved' IQ schizophrenia group

Table 2 Neurocognitive functioning in schizophrenia and bipolar disorder groups before and after controlling for IQ

\begin{tabular}{|c|c|c|c|c|c|c|c|c|c|c|c|c|c|c|}
\hline & \multicolumn{4}{|c|}{ Schizophrenia group } & \multicolumn{4}{|c|}{ Bipolar disorder group } & \multicolumn{6}{|c|}{ ANCOVA $^{a}$} \\
\hline & Mean & (s.d.) & $z^{\mathrm{a}}$ & $z^{\mathrm{b}}$ & Mean & (s.d.) & $z^{a}$ & $z^{\mathrm{b}}$ & $F^{\mathrm{a}}$ & $F^{\mathrm{b}}$ & d.f. $^{a}$ & d.f. ${ }^{\text {b }}$ & $P^{a}$ & $P^{\mathrm{b}}$ \\
\hline Verbal attention & 6.74 & $(1.06)$ & -0.27 & -0.01 & 6.69 & $(0.82)$ & -0.33 & -0.21 & 1.67 & 0.61 & 2,142 & 2,141 & NS & NS \\
\hline Visual attention & 4.90 & (1.09) & $-0.59 *$ & -0.4 & 4.92 & $(0.85)$ & -0.32 & -0.26 & 3.8 & 1.3 & 2,141 & 2,140 & 0.05 & NS \\
\hline Working memory & 3.94 & $(1.10)$ & $-1.05^{\star \star}$ & $-0.59 * *$ & 4.38 & $(1.31)$ & $-0.69^{\star *}$ & $-0.50^{\star}$ & 16.19 & 5.26 & 2,142 & 2,141 & 0.001 & 0.01 \\
\hline Verbal memory & 12.81 & (4.32) & $-1.21 * \star$ & $-0.78^{* \star}$ & 12.88 & $(4.52)$ & $-0.85^{\star *}$ & $-0.71 * *$ & 18.4 & 7.72 & 2,141 & 2,140 & 0.001 & 0.001 \\
\hline Visual memory & 11.76 & (5.73) & $-1.27^{\star \star}$ & $-0.81 * \star$ & 11.78 & $(6.50)$ & $-1.14^{\star *}$ & $-1.04^{\star *}$ & 27.53 & 14.6 & 2,141 & 2,140 & 0.001 & 0.001 \\
\hline Response inhibition ${ }^{c}$ & 23.42 & $(19.19)$ & $-1.4^{* \star}$ & $-0.90 * *$ & 17.69 & $(17.50)$ & $-0.68^{* *}$ & $-0.52^{\star}$ & 18.23 & 6.48 & 1,141 & 2,140 & 0.001 & 0.01 \\
\hline Executive set-shifting & 19.74 & $(8.32)$ & $-1.35^{\star *}$ & -0.80 ** & 20.28 & $(6.96)$ & $-1.14^{\star *}$ & $-0.96 * *$ & 19.88 & 8.93 & 1,141 & 2,140 & 0.001 & 0.001 \\
\hline Verbal fluency ${ }^{c}$ & 9.56 & (3.81) & $-1.13^{\star *}$ & $-0.50^{*}$ & 11.57 & $(3.07)$ & -0.41 & -0.18 & 13.78 & 2.61 & 1,142 & 2,141 & 0.001 & 0.08 \\
\hline $\begin{array}{l}\text { Phonological } \\
\text { processing }\end{array}$ & 8.56 & $(6.48)$ & $-1.34^{\star \star}$ & $-0.76^{\star *}$ & 8.86 & $(6.50)$ & $-1.18^{\star *}$ & $-0.98^{* *}$ & 17.1 & 7.5 & 1,141 & 2,140 & 0.001 & 0.001 \\
\hline Callosal function ${ }^{c}$ & 21.40 & (7.77) & $-1.1^{* *}$ & $-0.47^{\star}$ & 23.80 & $(7.85)$ & -0.38 & -0.31 & 10.48 & 1.91 & 1,141 & 2,140 & 0.001 & NS \\
\hline \multicolumn{15}{|c|}{$\begin{array}{l}\text { NS, not significant. } \\
\text { a. Main effect of group reported: no covariate, d.f. }=142 \text {; age covariate, d.f. }=141 \text {. } \\
\text { b. Main effect of group reported: IQ covariate, d.f. }=141 \text {; age and IQ covariate, d.f. }=140 \text {. } \\
\text { C. Measures on which bipolar disorder group could be significantly differentiated from schizophrenia group, before controlling for IQ. } \\
\text { *Significantly different from controls, } P<0.05 \text {. } \\
\text { **Significantly different from controls, } P<0.01 \text {. }\end{array}$} \\
\hline
\end{tabular}


and bipolar disorder group) were verbal response inhibition $(P<0.01)$ and verbal fluency $(P<0.01)$, i.e. two of the three domains found to differentiate schizophrenia from bipolar disorder in the primary analyses. They were also more impaired (trend) on measures of working memory, verbal memory and premorbid IQ $(P<0.1)$ compared with the bipolar disorder group and the 'preserved IQ' schizophrenia group, and had significantly poorer callosal functioning compared with the bipolar disorder group $(P<0.05)$, i.e. the third domain on which schizophrenia could be differentiated from bipolar disorder in initial ANCOVAs. Notably, all three of these groups performed similarly on measures of attention, visual memory, executive functioning and language.

Post hoc analyses clearly indicated that factors other than diagnosis could account for the initial neurocognitive differences observed between the schizophrenia and bipolar disorder groups. In order to quantify the unique contributions these factors made to the differences observed, all variables under consideration (i.e. diagnosis, negative symptoms, depressive symptoms, age, current IQ) were entered into multiple linear regression models to predict response inhibition, verbal fluency and callosal function respectively. Results are shown in Table 3.

Predictors of poorer response inhibition were lower IQ, older age at testing and higher levels of negative symptoms. Predictors of impaired verbal fluency were lower IQ and higher levels of negative symptoms. Only lower IQ predicted poorer callosal functioning.

\section{Discussion}

In this paper, cognition was profiled and compared in participants with a first episode of bipolar disorder or schizophrenia. We attempted to determine the extent to which neurocognitive deficits are specific or generalised in those with bipolar disorder and schizophrenia. The schizophrenia group's profile of impairments was relatively non-specific in the primary analyses, replicating many previous observations made in first-episode schizophrenia cohorts. ${ }^{3,22-27}$ Schizophrenia-group participants were impaired on nine of ten measures relative to controls and were significantly poorer than the bipolar disorder group on measures of verbal fluency, verbal response inhibition and callosal functioning. However, when global cognitive functioning was controlled for, the schizophrenia group could no longer be discriminated from the bipolar disorder group on any measure. Secondary analyses also showed that participants with schizophrenia with a 'preserved IQ' did not present with a non-specific pattern despite high levels of negative symptoms: they performed similarly to the bipolar disorder group on all measures. Furthermore, differences in bipolar disorder $v$. 'low IQ' schizophrenia comparisons were roughly equivalent to those observed when 'preserved IQ' schizophrenia was compared with 'low IQ' schizophrenia. Collectively, these observations suggest that the diagnostic differences observed between these psychosis groups on selective measures of neurocognitive functioning are best understood with reference to the greater propensity of people with schizophrenia to experience general cognitive impairment. Lower IQ in those with schizophrenia is associated with a greater quantitative downward shift in certain neurocognitive domains (namely, in the domains of verbal fluency, response inhibition and callosal functioning). By extension, the lower the general intellectual functioning of a given participant, the more likely they were to present with a relatively non-specific deficit pattern, rendering the search for differential deficits in specific neurocognitive functions difficult in the schizophrenia group and futile in those with schizophrenia and 'low' IQ. Floor effects for the tests, a greater preponderance of specific negative symptoms in the 'low IQ' schizophrenia group such as loss of motivation, and/or greater difficulty understanding neurocognitive test instructions are some of the potential contributors to the latter observation.

\section{Selective and general cognitive impairments}

The non-specific profile of multiple cognitive deficits observed in schizophrenia has been the subject of considerable debate in the literature. ${ }^{22,23}$ It has been suggested that a non-specific deficit pattern is indicative of either diffuse dysfunction in cortical circuitry or disturbances in key systems that modulate broadly distributed neural networks. ${ }^{22}$ Comparisons between the bipolar disorder group, the schizophrenia 'preserved' and 'low' IQ groups

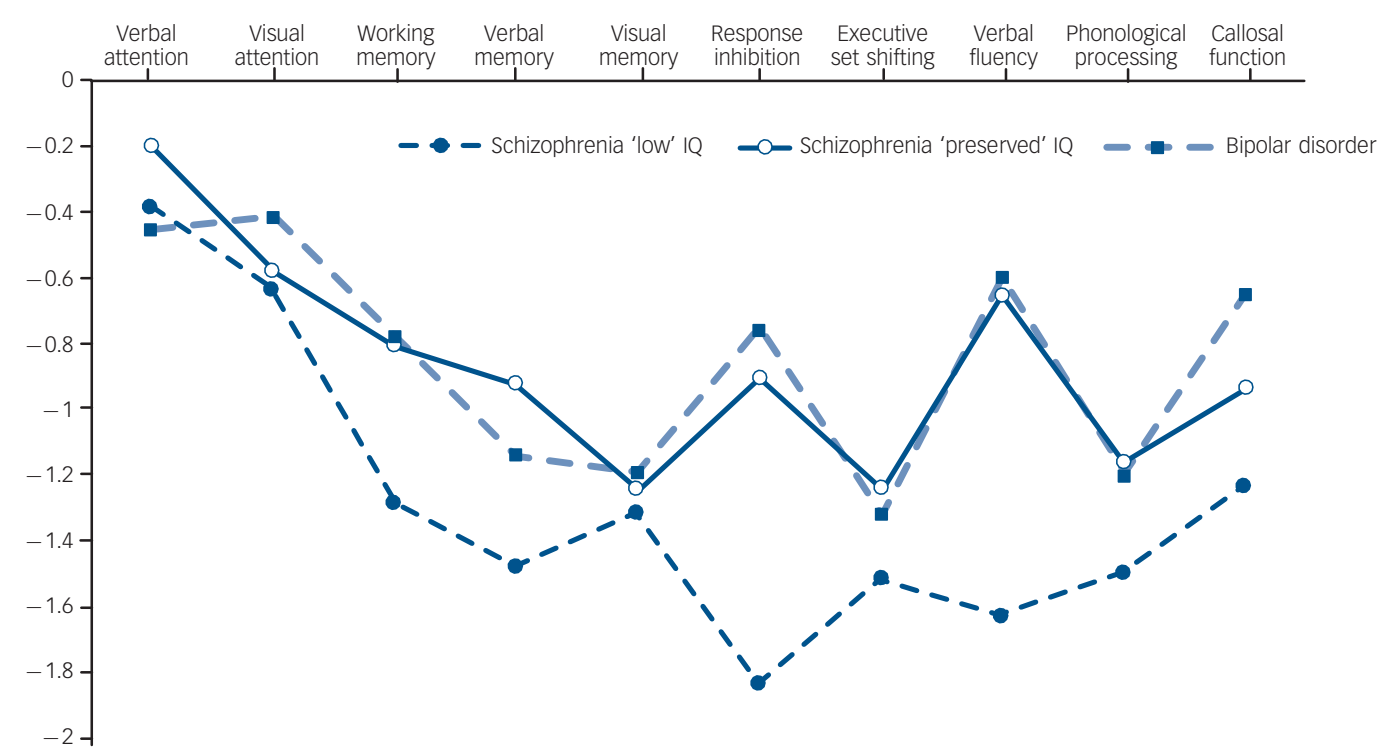

Fig. 1 Neurocognitive profile of schizophrenia groups with 'preserved' and 'low' IQ relative to bipolar disorder group and controls. Controls z-scores: mean $=0$ (s.d. $=1$ ). 


\begin{tabular}{|c|c|c|c|}
\hline & Response inhibition & Verbal fluency & Callosal function \\
\hline Adjusted $\mathrm{R}^{2}(P)$ & $0.28(<0.001)$ & $0.33(<0.001)$ & $0.12(<0.05)$ \\
\hline Diagnosis (schizophrenia/bipolar disorder), $\beta(P)$ & $-0.04(\mathrm{NS})$ & $-0.03(\mathrm{NS})$ & $0.02(\mathrm{NS})$ \\
\hline Positive and Negative Syndrome Scale: total negative, $\beta(P)$ & $-0.24(0.08)$ & $-0.27(<0.05)$ & $0.01(\mathrm{NS})$ \\
\hline Beck Depression Inventory, $\beta(P)$ & $-0.05(\mathrm{NS})$ & $-0.02(N S)$ & -0.09 (NS) \\
\hline Age, $\beta(P)$ & $-0.25(<0.05)$ & $-0.01(\mathrm{NS})$ & -0.04 (NS) \\
\hline Wechsler Abbreviated Scale of Intelligence IQ, $\beta(P)$ & $0.4(<0.001)$ & $0.47(<0.001)$ & $0.36(<0.01)$ \\
\hline
\end{tabular}

contribute to this debate. The bipolar disorder group had impairments in many selective domains of neurocognitive function against a background of preserved general cognitive functioning. The shape of their cognitive profile was also very similar to that of the 'preserved IQ' schizophrenia group. The most pronounced deficits in both bipolar disorder and schizophrenia were in visual and verbal memory, executive set shifting and language functioning. These clinically significant selective deficits in neurocognitive function do not appear to be artefacts of a global cognitive impairment or negative symptoms. Rather these particular deficits possibly underlie psychosis per se, reflecting abnormalities in a common pathway at a systems level that is evident early in the course of the psychoses. Their utility as putative endophenotypes may not be specific to schizophrenia and it is reasonable to argue that cohorts of people with psychosis could be examined as a single set of interest to improve statistical power in genetic studies that make use of these endophenotypes.

However, as the majority of participants were unwell at the time of testing, it is essential to examine these neurocognitive domains longitudinally in the NIFEPS cohort to determine the extent to which these are 'trait' or 'state' deficits. It might be hypothesised that the group with bipolar disorder examined in this study showed a fair degree of normalisation in cognition as they became progressively euthymic. Of particular interest would be comparisons between individuals with bipolar disorder and those individuals with 'preserved IQ' and schizophrenia following a more lengthy period of treatment and improvements in symptoms. It is possible that what makes schizophrenia unique from a neurocognitive perspective is not the precise pattern or magnitude of selective deficits observed cross-sectionally, but the failure to improve on specific selective domains with the resolution of psychotic symptoms regardless of whether IQ is 'preserved' at first episode or not.

\section{Implications for the use of diagnostic criteria}

A related issue is how these data affect the use of diagnostic classification in neurocognitive research of the psychoses. It could be tentatively argued with reference to the limitation outlined above that people with schizophrenia and a 'preserved' IQ are more suitably grouped with people with bipolar disorder, or alternatively, that individuals with psychosis should be divided into separate groups according to whether they fall above or below 1 standard deviation of the expected mean IQ for the general population for example. Comparing 'preserved IQ' diagnostic groups, or clustering these 'preserved IQ' participants according to specific symptoms for comparative purposes, may shed greater light on how the severity of positive, affective and, potentially, negative symptoms contribute to selective neurocognitive impairments and functional outcome in people with psychosis in the absence of the highly pervasive influence of low IQ within a cohort. Given recent meta-analyses ${ }^{28,29}$ that identify impairments in people with euthymic bipolar disorder in the domains of attention, executive functioning and memory, it is possible that the existing literature on neurocognitive functioning in individuals with bipolar disorder may prove of some relevance to individuals with schizophrenia and a relatively preserved IQ. It might also be speculated that grouping people with psychosis according to 'preserved' and 'low' IQ may be of particular use when prospectively conducting a comparative examination of the longitudinal course of the psychoses, and when retrospectively examining aspects of the neurodevelopmental hypothesis of schizophrenia. However, the issues of 'potential' v. 'achieved' IQ of people with schizophrenia in adolescence, and the decline in IQ that can occur following the onset of illness, would need to be carefully considered before adopting these approaches.

\section{Confounding effects of medication}

A second limitation to the conclusions from these analyses is the potential confounding effects of medication. Given the fact that almost all of the participants were medicated at the time of testing, most receiving antipsychotics, it was not possible to fully gauge the contribution this factor made to their neurocognitive functioning. Furthermore, medications may have influenced the neurocognitive performance of the schizophrenia and bipolar disorder groups differently. Undoubtedly, such issues can only be addressed through controlled clinical trials, and studies examining cognition in individuals who have never received medication. However, the latter have consistently demonstrated that people who are antipsychotic naive show similar impairments to those on medication. Brickman et al ${ }^{24}$ compared 29 nevermedicated adolescents with a first-episode psychosis with 17 matched controls and reported that those with psychosis significantly underperformed on all measures, but were most impaired on tests of memory, attention, and executive functioning. Saykin and colleagues ${ }^{25}$ also reported that antipsychotic-naive patients with a schizophrenia-spectrum first-episode psychosis $(n=37)$ had a near identical profile of neurocognitive deficits to previously treated but currently unmedicated individuals with schizophrenia $(n=67)$, both groups showing generalised impairment and selective deficits in attention, memory and executive functioning. Similar conclusions were also reached by Hill et $a l^{3}$ in a large study that compared participants with a schizophrenia-spectrum first-episode psychosis $(n=86)$ with participants with a first episode of psychotic depression $(n=14)$ and healthy volunteers $(n=81)$. Medication is therefore highly unlikely to account for the neuropsychological deficits observed in our study of people with first-episode psychosis.

\section{Stability of diagnoses}

A third limitation relates to the stability of individuals' diagnoses. It is recognised that diagnosing people during their first episode is difficult and it is possible that individuals classified as having a bipolar disorder may change to schizophrenia (and, although less 
likely, vice versa). Paradoxically, this, as well as problems related to the availability of different diagnostic classification systems, forms part of the rationale for abandoning diagnosis as a variable in research studies. In this study, stability of diagnoses was good at 1 year as determined by operational criteria (OPCRIT) $)^{30}$ ICD-10: diagnostic stability was $89 \%$ for the schizophrenia group ( $0 \%$ switch to bipolar disorder, $5 \%$ switch to psychotic depression) and $72 \%$ for the bipolar disorder group (16\% switch to schizophrenia), which is similar or better than that reported in the literature. ${ }^{31,32}$

\section{Implications for research and practice}

Overall, this study demonstrates that neurocognitive impairments in memory, executive functioning and language are common to people with first-episode psychosis and with further study, these neurocognitive impairments may prove useful as endophenotypic markers for psychosis in general. Furthermore, consideration of the degree of global cognitive impairment within cohorts is critical when attempting to understand both diagnostic differences between psychosis groups and patterns of selective neurocognitive impairment in people with schizophrenia.

Suzanne L. Barrett, PhD, School of Psychology, University of Ulster Jordanstown, Co. Antrim, Northern Ireland; Ciaran C. Mulholland, MD, MRCPsych, Stephen J. Cooper, MD, FRCPsych, Division of Psychiatry and Neuroscience, Queen's University Belfast, Northern Ireland; Teresa M. Rushe, PhD, School of Psychological Sciences, University of Manchester, UK

Correspondence: Suzanne Barrett, Division of Psychiatry and Neuroscience, Queens University Belfast, Whitla Medical Building, 97 Lisburn Road, Belfast BT9 7BL, UK. Email: s.I.barrett@qub.ac.uk

First received 15 May 2008, final revision 16 Oct 2008, accepted 12 Jan 2009

\section{Funding}

Funded by the Research and Development Office (R \& D Office), Northern Ireland.

\section{Acknowledgement}

The authors gratefully acknowledge the assistance of psychiatrists and clinical teams working in Belfast and Northern Health and Social Care Trusts, Northern Ireland.

\section{References}

1 Gottesman II, Gould TD. The endophenotype concept in psychiatry. Etymology and strategic intentions. Am J Psychiatry 2003; 160: 636-45.

2 McClellan J, Prezbindowski A, Breiger D, McCurry C. Neuropsychological functioning in early onset psychotic disorders. Schizophr Res 2004; 68: 21-6.

3 Hill SK, Keshavan MS, Thase ME, Sweeney JA. Neuropsychological dysfunction in antipsychotic-naive first-episode unipolar psychotic depression. Am J Psychiatry 2004; 161: 996-1003.

4 Craddock N, O'Donovan MC, Owen MJ. Genes for schizophrenia and bipolar disorder? implications for psychiatric nosology. Schizophr Bull 2006; 32: 9-16.

5 Torrey EF. Epidemiological comparison of schizophrenia and bipolar disorder Schizophr Res 1999; 39: 101-6.

6 World Health Organization. The ICD-10 Classification of Mental and Behavioural Disorders: Clinical Descriptions and Diagnostic Guidelines. WHO 1992.
7 Wing JK, Babor T, Brugha T, Burke J, Cooper JE, Giel R, et al. SCAN. Schedules for Clinical Assessment in Neuropsychiatry. Arch Gen Psychiatry 1990; 47: 589-93.

8 Bebbington P, Nayani T. The Psychosis Screening Questionnaire. Int J Methods Psychiatr Res 1995; 5: 11-9.

9 Kay SR, Opler LA, Lindenmayer JP. Reliability and validity of the positive and negative syndrome scale for schizophrenics. Psychiatry Res 1988; 23: 99-110.

10 Beck AT, Steer A, Brown GK. Beck Depression Inventory Manual, (4th edn). Psychological Corporation, 1996.

11 Wechsler D. Wechsler Memory Scale (3rd edn) (WMS-III). Harcourt Assessment, 1997.

12 Nelson HE. National Adult Reading Test (NART): Test Manual. nferNelson, 1982

13 Wechsler D. Wechsler Abbreviated Scale of Intelligence. Psychological Corporation, 1999.

14 Milner B. Interhemispheric differences in the localization of psychological processes in man. Br Med Bull 1971; 27: 272-7.

15 Meyers JE, Meyers KR. Rey Complex Figure Test and Recognition Trial. Professional Manual. Psychological Assessment Resources, 1995.

16 Burgess $\mathrm{P}$, Shallice T. The Hayling and Brixton Tests. Test Manual. Thames Valley Test Company, 1997.

17 Lezak MD, Howieson DB, Loring DW, Hannay HJ, Fischer JS Neuropsychological Assessment. Oxford University Press, 2004.

18 Perin D. Phonemic segmentation and spelling. Br J Psychol 1983; 74: 129-44.

19 Satomi K, Kinoshita Y, Hirakawa S. Disturbances of cross-localization of fingertips in a callosal patient. Cortex 1991; 27: 327-31.

20 Schafer JL. Multiple imputation: a primer. Stat Methods Med Res 1999; 8: 3-15.

21 Lindenmayer JP, Brown E, Baker RW, Schuh LM, Shao L, Tohen M, et al. An excitement subscale of the Positive and Negative Syndrome Scale. Schizophr Res 2004; 68: 331-7.

22 Mohamed S, Paulsen JS, O'Leary D, Arndt S, Andreasen N. Generalized cognitive deficits in schizophrenia. A study of first-episode patients. Arch Gen Psychiatry 1999; 56: 749-54.

23 Bilder RM, Goldman RS, Robinson D, Reiter G, Bell L, Bates JA, et al. Neuropsychology of first-episode schizophrenia. Initial characterization and clinical correlates. Am J Psychiatry 2000; 157: 549-59.

24 Brickman AM, Buchsbaum MS, Bloom R, Bokhoven P, Paul-Odouard R, Haznedar MM, et al. Neuropsychological functioning in first-break, nevermedicated adolescents with psychosis. J Nerv Ment Dis 2004; 192: 615-22.

25 Saykin AJ, Shtasel DL, Gur RE, Kester DB, Mozley LH, Stafiniak P, et al. Neuropsychological deficits in neuroleptic naive patients with first-episode schizophrenia. Arch Gen Psychiatry 1994; 51: 124-31.

26 Riley EM, McGovern D, Mockler D, Doku VC, OCeallaigh S, Fannon DG, et al. Neuropsychological functioning in first-episode psychosis - evidence of specific deficits. Schizophr Res 2000; 43: 47-55.

27 Fitzgerald D, Lucas S, Redoblado MA, Winter V, Brennan J, Anderson J, et al. Cognitive functioning in young people with first episode psychosis. Relationship to diagnosis and clinical characteristics. Aust N Z J Psychiatry 2004; 38: 501-10.

28 Robinson $\mathrm{U}$, Thompson JM, Gallagher $\mathrm{P}$, Goswami U, Young $\mathrm{AH}$, Ferrier IN et al. A meta-analysis of cognitive deficits in euthymic patients with bipolar disorder. J Affect Disord 2006; 93: 105-15.

29 Torres IJ, Boudreau VG, Yatham LN. Neuropsychological functioning in euthymic bipolar disorder: a meta-analysis. Acta Psychiatr Scand Suppl 2007; 434: 17-26.

30 McGuffin P, Farmer A, Harvey I. A polydiagnostic application of operational criteria in studies of psychotic illness. Arch Gen Psychiatry 1999; 48: 764-70.

31 Chen YR, Swann AC, Johnson BA. Stability of diagnosis in bipolar disorder. J Nerv Ment Dis 1998; 186: 17-23.

32 Mihalopoulos $C$, McGorry $P$, Roberts $S$, McFarlane $C$. The procedural validity of retrospective case note diagnosis. Aust N Z J Psychiatry 2000; 34: 154-9. 